\title{
Repetitive TMS over the left dorsolateral prefrontal cortex modulates the error positivity: An ERP study
}

\author{
Fabio Masina ${ }^{\mathrm{a}, \mathrm{b}, *, 1}$, Vincenza Tarantino ${ }^{\mathrm{c}, \mathrm{d}, 1}$, Antonino Vallesi ${ }^{\mathrm{c}, \mathrm{e}}$, Daniela Mapelli ${ }^{\mathrm{a}, \mathrm{b}}$ \\ ${ }^{a}$ Department of General Psychology, University of Padova, Italy \\ ${ }^{\mathrm{b}}$ Human Inspired Technologies Research Center, University of Padova, Italy \\ ${ }^{\mathrm{c}}$ Department of Neuroscience \& Padova Neuroscience Center, University of Padova, Italy \\ ${ }^{\mathrm{d}}$ Department of Psychology, Educational Science and Human Movement, University of Palermo, Italy \\ ${ }^{\mathrm{e}}$ Brain Imaging and Neural Dynamics Research Group, IRCCS San Camillo Hospital, Venice, Italy
}

\section{A R T I C L E I N F O}

\section{Keywords:}

Error awareness

Post-error slowing (PES)

Transcranial magnetic stimulation (TMS)

Error positivity (Pe)

Error-related negativity (ERN)

Dorsolateral prefrontal cortex (DLPFC)

\begin{abstract}
A B S T R A C T
Error processing is a critical step towards an efficient adaptation of our behavior to achieve a goal. Little research has been devoted to investigate the contribution of the dorsolateral prefrontal cortex (DLPFC) in supporting error processing. In this study, the causal relationship of the DLPFC in error commission was examined by means of a repetitive transcranial magnetic stimulation protocol (rTMS). Specifically, the effects of an inhibitory protocol were assessed by examining the electroencephalographic signal recorded during the execution of a Go/ No-Go task. To this aim, a group of 15 healthy young participants performed a three-session study. At each session, either the right DLPFC, the left DLPFC, or the Vertex (control site) were stimulated, for $20 \mathrm{~min}$ at $1 \mathrm{~Hz}$. Immediately after the stimulation, participants performed the task. Although no behavioral effects of rTMS emerged, the analysis of event-related electric potentials (ERPs) revealed that the amplitude of a positive potential evoked by error commission, the error positivity (Pe), was reduced after the stimulation of the left DLPFC. On the contrary, the earlier error-related negativity component (ERN) was not affected. These results revealed that the left DLPFC intervenes at later stages of error-related processes. We could speculate that its role is specifically linked to error awareness.
\end{abstract}

\section{Introduction}

In everyday life, minor errors are ordinary events that do not generally produce significant effects. However, also a minor error can have important consequences, for example, a driving distraction. Thus, it is not surprising that human error is extensively studied.

Investigations that examine human error can be classified in: (1) studies focused on antecedents of an error, namely on the causes of the error, and (2) studies interested in the consequences of an error, namely the effects that an error produces on a system or the reaction of a system following an error. An interesting phenomenon, related to this latter research line, concerns post-error slowing (PES), already described in 1966 by Rabbitt and colleagues. PES reflects a general individual tendency to slow down a motor response on the next trial after committing an error (Danielmeier and Ullsperger, 2011; Ullsperger et al., 2014). Some authors have interpreted this slowing as an increase in response caution, which allows individuals to maintain constant the level of accuracy and indexes cognitive control/monitoring strategies (e.g., Botvinick et al., 2001); according to other authors, PES reflects a behavioral impairment caused by an error (Notebaert et al., 2009). Another crucial error-related process is error awareness. The conscious detection of an error has an adaptive role in everyday life, since an aware error triggers a series of adjustments that allow correcting and avoiding the same error in the future (Laming, 1968; Ullsperger et al., 2014).

Along with behavioral indexes (PES and error awareness), a rich corpus of EEG studies has investigated the electrophysiological correlates of error-related processes. Two event-related potentials (ERPs) have been found as the most representative markers associated with the commission of errors. The first one, the error-related negativity (ERN) is a negative deflection that reaches maximal amplitude values at about 50-100 ms after the erroneous response. This ERP component has a frontocentral voltage distribution on the scalp (Falkenstein et al., 1991; Gehring et al., 1990). Following the ERN, a second positive deflection,

\footnotetext{
* Corresponding author. Department of General Psychology, University of Padova, Via Venezia 8, 35100 Padova, Italy.

E-mail addresses: fabio.masina@phd.unipd.it, fabio.masina@unipd.it (F. Masina).

${ }^{1}$ Both authors contributed equally to this work.
} 
called error positivity (Pe), emerges at about $200-400 \mathrm{~ms}$ after the erroneous response. The scalp distribution of the Pe shows a maximum at central sites (Falkenstein et al., 1991; Overbeek et al., 2005). The neural generator of the ERN has been localized in several brains regions, including the medial frontal regions of the cortex (Debener, 2005) and the supplementary motor area (Bonini et al., 2014). Functional magnetic resonance (fMRI) studies have shown that the ERN occurs in the anterior cingulate cortex (ACC; Iannaccone et al., 2015; Ito et al., 2003; Ullsperger and Von Cramon, 2001). Taken together, the heterogeneity of results suggest that the ERN has multiple neural generators (Kappenman and Luck, 2012). Similarly, studies attempting to pinpoint the neural generator of the Pe have found mixed results. Dipole source modeling of the Pe scalp distribution has suggested two possible regions candidate as neural generators of the Pe: the rostral (van Boxtel et al., 2005; Veen and Carter, 2002) and caudal (Herrmann et al., 2004) portion of the medial frontal cortex. Furthermore, an intracranial EEG study has shown the involvement of different regions that included the cingulate, mesiotemporal, orbitofrontal cortices and the dorsolateral prefrontal cortex (DLPFC; Brázdil et al., 2002).

From a functional point of view, the ERN and the Pe components have been found to be independent and to reflect different aspects of error processing (Coleman et al., 2018; Di Gregorio et al., 2018). The ERN has been related to a manifestation of an error, regardless of its conscious detection (Nieuwenhuis et al., 2001) and seems to reflect the activity of a system that detects a conflict between planned and executed actions. In other words, the ERN would reflect the monitoring of a cognitive mismatch (Falkenstein et al., 1991; Gehring et al., 1993; Holroyd and Coles, 2002). The Pe component has been associated with multiple functions, such as the awareness of an error (Endrass et al., 2007; Hoonakker et al., 2016; Murphy et al., 2012; O'Connell et al., 2007; Overbeek et al., 2005), the motivational significance attributed to an error (Ridderinkhof et al., 2009), the accumulation of evidence that an error has occurred (Steinhauser and Yeung, 2010), and the involvement of adaptive strategies following an error (Overbeek et al., 2005).

Although many studies have focused on understanding the effects of committing an error on behavior and brain activity, few studies have tried to directly modulate error-related processes, such as PES and error awareness. With regards to PES, Sellaro et al. (2015) observed an increase in PES in healthy young individuals by applying transcutaneous vagal nerve stimulation. Harty et al. (2014) increased error awareness by using anodal transcranial direct current stimulation (tDCS) on the right DLPFC in a group of older adults. Hester et al. (2012) enhanced error awareness by the administration of a single dose of methylphenidate, demonstrating a link between error awareness and the catecholaminergic neurotransmission. More recently, Masina et al. (2018) demonstrated that an on-line single-pulse transcranial magnetic stimulation protocol (TMS) delivered on the right and on the left DLPFC induced a reduction of error awareness in a group of healthy participants.

With regards to the ERN and the Pe, these ERP components have been modulated by noninvasive brain stimulations (NIBS) as well. In a tDCS study, Reinhart and Woodman (2014) demonstrated that the stimulation of the medial frontal cortex could eliminate or enhance the ERN, by administering cathodal or anodal tDCS, respectively. Bellaïche et al. (2013) found that cathodal tDCS over the medial frontal cortex reduced the Pe amplitude compared to both anodal and sham stimulation. Rollnik et al. (2004) modulated the ERN and Pe amplitude by means of a low-frequency $(0.9 \mathrm{~Hz})$ rTMS. Specifically, in this study, the TMS was delivered over medial frontal regions (including the ACC) and over lateral frontal regions (right DLPFC). Only the stimulation of the ACC provoked both a reduction of the ERN amplitude and an increase of the Pe amplitude. The authors suggested that the stimulation of medial frontal regions might have modulated a crucial area associated with the ERN, namely the ACC. However, it was unclear how these deep brain area could be stimulated by means of a conventional figure-of- 8 coil and the involvement of the left lateral prefrontal regions was not considered.

Apart from the mentioned NIBS studies, little evidence has established causal links between error-related processes and their brain correlates, especially because the brain correlates of error-related processes mainly derive from EEG and fMRI studies. These techniques, despite the fascinating perspective they provide, allow establishing only correlational inferences on the brain-cognition-behavior relation. The main purpose of the present study was to overcome the correlational approach of fMRI and EEG techniques and to examine the contribution of the DLPFC in error processing. To this aim a TMS protocol was performed, combined with behavioral and ERP measures. Namely, a low-frequency $(1-\mathrm{Hz})$ rTMS protocol was applied to young healthy volunteers with the aim of inhibiting processes associated with error commission. The 1-Hz rTMS protocol is known to reduce cortical excitability in targeted brain areas for several minutes after the end of stimulation (Chen et al., 1997). The selected target areas were the right and left DLPFC. As previously remarked, lateral prefrontal regions have been suggested to be involved in error awareness together with the well-known role of medial frontal cortex (Harty et al., 2014; Masina et al., 2018). Interestingly, a recent study showed a relationship between the left DLPFC and PES (Mansouri et al., 2016). Furthermore, neuropsychological findings supported an involvement of the DLPFC in the generation of the ERN (Gehring and Knight, 2000; Sokhadze et al., 2014, 2012). Wessel et al. (2014) proposed that the lateral prefrontal cortex is a crucial hub within the prefrontal-cingulate performancemonitoring network (PCMN), which triggers compensatory mechanisms following an error (e.g., PES). The DLPFC seems to be central in metacognitive processes (Qiu et al., 2018), such as error awareness (Hoerold et al., 2013; see also Harty et al., 2014; Masina et al., 2018). However, the causal contribution of dorsolateral prefrontal areas has not been elucidated yet. Moreover, compelling evidence about a functional hemispheric lateralization of the lateral prefrontal cortex in error-related processing has not been found yet, especially because the role of the right and left DLPFC was investigated in separate studies (Rollnik et al., 2004; Mansouri et al., 2016, respectively), but it has not been directly compared in the same experiment. In order to overcome this limitation, in the present study we investigated the contribution of both the right and left DLPFC. Our working hypothesis was that if the DLPFC contributes to processes related to error awareness, low-frequency inhibitory rTMS should produce fewer aware errors and decreased PES. At the same time, inhibitory stimulation of this region should induce an attenuation of the error-related potentials, namely the ERN if the DLPFC acts on earlier stage of error detection, the Pe if this area intervenes at later stages.

\section{Methods}

\subsection{Participants}

Fifteen right-handed healthy participants aged 20-34 (mean $=24.3$ years; $S D=3.6$ ) took part in the study (9 females). All participants had normal or corrected-to-normal visual acuity. Exclusion criteria were a history of neurological or psychiatric diseases, and use of neurological or psychiatric medications. Before experiment, participants gave their written informed consent and were checked for TMS exclusion criteria (Rossi et al., 2011). The adopted safety procedures were in line with the guidelines for the use of TMS (Rossi et al., 2009). The study was approved by the Ethics Committee of School of Psychology, University of Padua (protocol no. 1929). The experimental procedure was in accord with the ethical principles of the 1964 Declaration of Helsinki.

\section{2. rTMS paradigm}

Repetitive TMS was performed using a Magstim Rapid ${ }^{2}$ TMS stimulator (Magstim Company, Whitland, UK) with a 70-mm figure-of- 


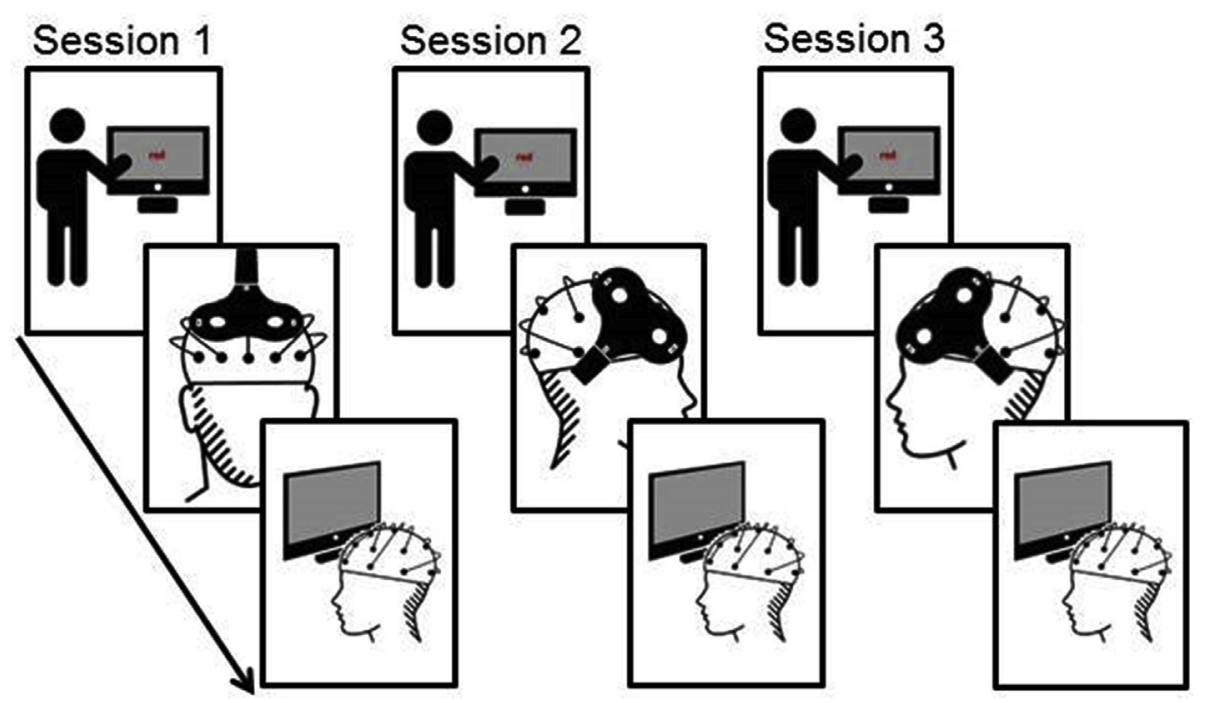

Fig. 1. An example of experimental session in which the order of the stimulation sites, namely the Vertex, the right DLPFC, and the left DLPFC, is depicted.

eight stimulation coil. As detailed in the procedure reported below, the stimulation targets were identified with Brainsight frameless stereotaxic system (Rogue Research, Montreal, Canada) and the position of the coil was maintained in real-time by the optical tracking system Polaris Vicra (NDI, Waterloo, Canada).

\subsection{EEG recording}

The EEG signal was recorded by means of $64 \mathrm{Ag} / \mathrm{AgCl}$ sintered ring electrodes mounted on an elastic cap according to the International 10-20 system (EASYCAP GmbH, Germany). Compared to standard electrodes, they are specifically designed to be compatible with simultaneous TMS, because they avoid overheating during magnetic stimulation and eliminate any risk for participants. Furthermore, their thickness is thinner than standard electrodes $(<4 \mathrm{~mm})$, reducing the space between the TMS coil and participant's scalp. The cap was connected to an AC amplifier (Micromed SD MRI, Micromed, Mogliano Veneto, Italy). The amplifier was optically connected to a PC and BrainQuick System Plus software allowed monitoring EEG during every session.

The EEG recordings were referenced to $\mathrm{FCz}$ electrode, while the ground electrode was placed on AFz. Raw data were recorded with a high-pass filter of $0.016 \mathrm{~Hz}$ and digitalized with a frequency of $512 \mathrm{~Hz}$.

\subsection{Task}

During EEG recording, participants performed an adapted version of the Error Awareness Task (EAT; Hester et al., 2005). In this task, a serial stream of single color words was presented at the center of the screen on a gray background. Participants were trained to respond with a single-speeded press (" 3 " on the keyboard), when the word and its color form were congruent (Go trials). They were asked to withhold this response when the word and its color font were incongruent (Stroop NoGo trials), or when the word was presented in two consecutive trials (repeat No-Go trials). In case participants failed to withhold their responses in either No-Go conditions (Stroop and repeat), they were instructed to signal as soon as possible the commission error by pressing a different button (the space bar). In order to maintain the number of errors between participants as similar as possible, we adopted an adaptive staircase approach. Specifically, the task difficulty was based on the participants' accuracy on No-Go trials. We manipulated the duration of ISI so that the time required for maintaining the previous word in memory was modified as a function of accuracy. The ISI length was extended to induce more commission errors on repeat trials, in fact, participants had to retain for a longer time the stimulus in working memory. At the beginning of the task, the words were presented for $200 \mathrm{~ms}$ with an ISI of $1800 \mathrm{~ms}$. ISI durations could change according to three scenarios: (1) if the accuracy was below $50 \%$, the ISI duration was set at $1700 \mathrm{~ms}$; (2) if the accuracy was higher than $60 \%$, the ISI duration was set at $2000 \mathrm{~ms}$; (3) if the accuracy was between $50 \%$ and $60 \%$, the ISI duration was set at $1800 \mathrm{~ms}$, as at the beginning of the task. The accuracy percentage was computed and updated after each No-Go trial. The task was divided into two equal blocks and each block lasted $10 \mathrm{~min}$. The total number of trials in the task was 668 , specifically 468 Go trials, 100 repeat No-Go trials and 100 Stroop No-Go trials. Participants rested their head on a table-mounted head-rest which fixed their distance at $60 \mathrm{~cm}$ from a 19-inch monitor for the whole duration of the task. Stimulus presentation was controlled by E-Prime software (Psychological Software Tools, Pittsburgh, PA, USA; version 2.0.8.90).

\subsection{Procedure}

Each participant was involved in three experimental sessions, carried out on different days. In each session a different brain site was stimulated. In order to ensure an appropriate washout period, at least $24 \mathrm{~h}$ had to pass in between two consecutive sessions, with a range of days left between each session of 1-36 days. The stimulation sites were the right DLPFC, the left DLPFC, and the Vertex (control site). The order of the stimulation sites was randomly assigned to each participant, in order to control for practice and fatigue effects or at least to avoid systematic influences of these effects in our experimental design. Overall, each session was divided into three phases: (1) task training; (2) EEG cap placement and rTMS; (3) task during the EEG recording (Fig. 1).

In the first phase (task training), it was ensured that all participants fully understood the instructions of the task and familiarized with it. In this phase, participants performed a short version of the task that lasted 5 min. Five TMS pulses were delivered on Vertex so that participants could experience the somatic sensation and the clicking sound that TMS produces.

In the second phase (EEG placement and rTMS), the EEG cap was placed on participants' head. Electrode impedances were kept below $5 \mathrm{k}$ $\Omega$. Then, the participants' resting motor threshold (RMT) was determined, in line with the standardized procedure (Rossi et al., 2009).

Afterward, the stimulation targets were identified with Brainsight frameless stereotaxic system (Rogue Research, Montreal, Canada) and spatial transformation was used to adjust the MRI template (the nonlinear ICBM-152 template by the Montreal Neurological Institute, MNI) 
to individual head shapes. According to Cieslik et al. (2013), the MNI coordinates of the right DLPFC were $30,43,23$, whereas the MNI coordinates of the left DLPFC were $-30,43,23$. These MNI coordinates were selected because, in a previous study, Masina et al. (2018) identified that these coordinates were causally associated with two brain areas involved in error awareness, namely the right and left DLPFC. The position of the Vertex was the $\mathrm{Cz}$ site of the International 10-20 system.

Finally, rTMS was administered over the stimulation site randomly chosen for that session. Stimulation parameters were the following: frequency of $1 \mathrm{~Hz}, 0.1-\mathrm{ms}$ pulse duration, and field intensity of $90 \%$ of RMT. In total, in each session, 1200 TMS pulses were delivered for 20 min. During the stimulation, the coil was oriented with the handle at $45^{\circ}$ to the mid-sagittal line, when the right and left DLPFC were stimulated. In the Vertex session, the coil was positioned with the handle pointing backwards parallel with the midline. Immediately after the end of the stimulation, the EEG was recorded while participants performed the task (third phase).

\subsection{Behavioral analyses}

Participant's performance was evaluated in terms of response times (RTs), accuracy, error awareness, and PES. RTs below $100 \mathrm{~ms}$ were removed from analyses. All RTs above 2.5 standard deviation $(S D)$ from the mean of each participant were excluded and a logarithm transformation was applied on the remaining RTs, in order to improve normalization. Unaware errors were excluded from the analyses of PES ( $<3 \%$ of total No-Go trials).

Accuracy and RTs - Accuracy (withholding accuracy) was analyzed by a repeated measures $2 \times 3$ ANOVA with trial type (Stroop vs. repeat) and stimulation site (right DLPFC, left DLPFC, and Vertex) as withinsubject factors. In addition, a repeated measures $2 \times 3$ ANOVA was performed on mean RTs, with response type (correct vs. error) and stimulation site (right DLPFC, left DLPFC, and Vertex) as within-subject factors.

Error awareness - Mean error awareness was calculated as the percentage of correctly signaled commission errors on the total number of commission errors (O'Connell et al., 2009). Error awareness for commission errors on Stroop and repeat trials was computed separately since previous studies using the EAT have found higher error awareness for Stroop compared with repeat errors (Harty et al., 2014; Hester et al., 2009; O'Connell et al., 2007). Therefore, a repeated measures $2 \times 3$ ANOVA with trial type (Stroop vs. repeat) and stimulation site (right DLPFC, left DLPFC, and Vertex) as within-subject factors was conducted.

PES - This index was computed according the Dutilh method, namely by the comparison between post-error RTs and the associated pre-error RTs (Dutilh et al., 2012). We performed a repeated measures $2 \times 3$ ANOVA with go trial position (post vs. pre No-Go trial) and stimulation site (right DLPFC, left DLPFC, and Vertex) as within-subject factors.

The Bonferroni correction was always applied to multiple post-hoc analyses and a corrected alpha-level of 0.05 was considered. Finally, effect sizes were estimated by partial eta squared $\left(\eta_{\mathrm{p}}^{2}\right)$.

\subsection{ERP analyses}

Data were offline analyzed using custom routines in EEGLAB v14 (Delorme and Makeig, 2004) running on Matlab R2017b (The Mathworks Natic, MA, USA). The continuous EEG trace was first filtered with a windowed sinc FIR filter, with a cut-off frequency of $40 \mathrm{~Hz}$, a Kaiser Window type with a beta of 5.65, a maximum passband deviation of 0.001 and a transition band of $20 \mathrm{~Hz}$ (Widmann et al., 2015). Epochs from $200 \mathrm{~ms}$ before response (button press) and $500 \mathrm{~ms}$ after the response were extracted from the continuous EEG signal. Artifacts (eye blinks and muscle activity) were identified and removed by means of independent component analysis (ICA). To this aim, all independent components (ICs) were visually inspected in terms of scalp distribution, frequency, timing and amplitude (Chaumon et al., 2015). The mean number of removed ICs was $16.2(S D=5.3)$. Epochs contaminated by artifacts were further identified with an automatic procedure (Delorme et al., 2007). The following criteria were applied: i) $-100 /+100 \mu \mathrm{V}$ as extreme amplitude values threshold; ii) drifts larger than $\pm 50 \mu \mathrm{V}$ and R2 $>0.3$ for the linear trend test; iii) $S D>7$ (for each channel) and $S D>4$ (for all channels) from the mean probability distribution of amplitude values across epochs for the improbability test; iv) $S D>7$ (for each channel) and $S D>4$ (for all channels) from the kurtosis of the probability distribution. Epochs containing data points exceeding these criteria were excluded from analyses. In addition, epochs containing unaware errors were excluded because they were too rare to be included in ERP analysis (mean $=4.5 ; S D=3.6$ ). Baseline correction was performed by subtracting the mean voltage of a $100-\mathrm{ms}$ window (from 200 to $100 \mathrm{~ms}$ before response, i.e., button press) from each time point. Lastly, the ERP signal was re-referenced to the mean of all channels, excluding TP9 and TP10, and FCz was derived.

At the end of all preprocessing steps, epochs locked to incorrect button presses (i.e., aware No-Go errors) were extracted. Since at least 5 epochs for each stimulation site survived, the ERN and Pe could be quantified (Niessen et al., 2017; Olvet and Hajcak, 2008; Pontifex et al., 2010). The mean number of epochs included in the analyses was 22.3 $(S D=8.8)$ for the right DLPFC stimulation session, $27.4(S D=10)$ for the left DLPFC stimulation session, and $24.1(S D=10.1)$ for the Vertex stimulation session. The mean number of epochs did not differ across sessions $(F(2,28)=2.34, p=0.114)$, therefore we could proceed with analyses.

The visual inspection of error-locked grand-averaged ERPs revealed the presence of the two typical error-related components: a negative deflection over fronto-central sites, which reached maximal amplitude values immediately before an erroneous button press, followed by a larger positive deflection over central sites. These deflections were very attenuated or even absent in epochs containing correct Go responses (Fig. 2). Given all these characteristics, the two potentials represented the ERN (Falkenstein et al., 1991; Gehring et al., 1990), which sometimes has been reported shortly before the response (Gehring et al., 1993), and the Pe component (Falkenstein et al., 1991; Overbeek et al., 2005), respectively. According to previous literature (e.g., Falkenstein et al., 2000) and to grand-average inspection, we selected $\mathrm{FCz}$ and $\mathrm{Cz}$ electrodes for analyses, where error-related components were maximally expressed (Fig. 3). To capture significant amplitude modulations affected by the stimulation site, we performed point-by-point $t$-tests. Specifically, we selected two time windows, from -100 to $50 \mathrm{~ms}$ and $100-300 \mathrm{~ms}$, for examining amplitude modulations in the ERN and in the Pe component, respectively. To detect reliable across-session differences within these windows, the $t$-scores were corrected by a clusterbased permutation method using a family-wise alpha level of 0.05 (Groppe et al., 2011).

Error-related ERPs were further compared with correct-response ERPs. To this aim, epochs locked to correct Go-responses were extracted and amplitude quantified with the same procedure applied to errors. A total of 262.5 epochs $(S D=3.7)$ for the right stimulation, 262.5 $(S D=4.4)$ for the left stimulation, and $254.2(S D=4.1)$ for the Vertex were included in the analyses $(F(2,28)=0.95, p=0.40)$.

\section{Results}

\subsection{Behavioral results}

Accuracy and RTs - The mean accuracy and RTs for each trial type and response type are shown in Table 1 . The 2 (trial type) $\times 3$ (stimulation site) ANOVA on accuracy values did not yield significant results [main effect of stimulation site: $F(2,28)=1.95, p=0.161$, partial $\eta^{2}=0.12$; trial type $\times$ stimulation site interaction $F(2,28)=2.42$, $p=0.107$, partial $\left.\eta^{2}=0.15\right]$. The main effect of trial type showed a 

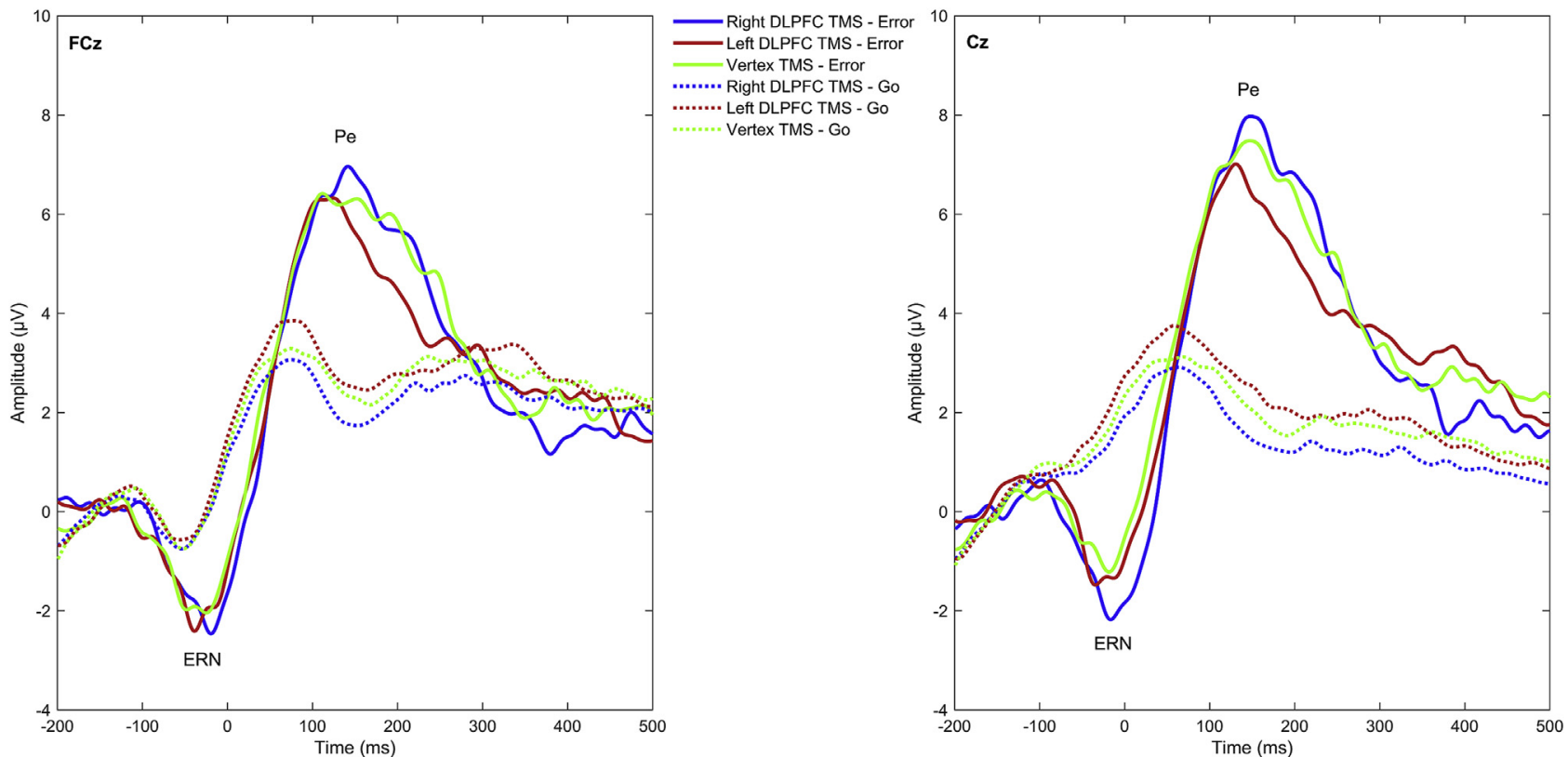

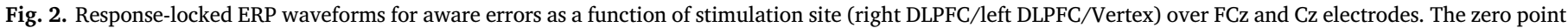
corresponds to incorrect button press.

tendency toward significance $(F(1,14)=4.34, p=0.056$, partial $\left.\eta^{2}=0.24\right)$, which revealed that participants were overall more accurate on repeat than on Stroop trials ( $87 \%$ vs. $82 \%$, respectively). The 2 (response type) $\times 3$ (stimulation site) ANOVA on RTs showed a main effect of response type $\left(F(1,14)=32.62, p<0.0001\right.$, partial $\left.\eta^{2}=0.70\right)$. Post-hoc comparisons showed that participants were faster when they committed an error than a correct response on Go trials $(516 \mathrm{~ms}$ vs. $544 \mathrm{~ms}$, respectively). A marginally significant effect of stimulation site emerged $\left(F(2,28)=3.203, p=0.056\right.$, partial $\left.\eta^{2}=0.19\right)$, which revealed slightly faster RTs when TMS was administered on the left than over the right sites $(p=0.073)$. No significant interaction was found $(F$ $(2,28)=0.664, p=0.523$, partial $\left.\eta^{2}=0.05\right)$.

Error awareness - The mean percentage of error awareness is presented in Table 1 . The 2 (trial type) $\times 3$ (stimulation site) ANOVA
Table 1

Mean and standard deviation $(S D)$ of performance on the EAT for right DLPFC, left DLPFC, and Vertex stimulation.

\begin{tabular}{llll}
\hline Measures & Right DLPFC & Left DLPFC & Vertex \\
\hline Stroop Accuracy (\%) & $85(10)$ & $79(10)$ & $82(8)$ \\
Repeat Accuracy (\%) & $88(7)$ & $88(5)$ & $86(10)$ \\
Stroop Awareness (\%) & $98(5)$ & $99(3)$ & $97(5)$ \\
Repeat Awareness (\%) & $71(22)$ & $69(21)$ & $72(30)$ \\
Go RT (ms) & $562(54)$ & $528(49)$ & $543(57)$ \\
Error RT (ms) & $534(55)$ & $507(61)$ & $508(60)$ \\
Post-error RT (ms) & $551(56)$ & $501(67)$ & $527(72)$ \\
Pre-error RT (ms) & $558(51)$ & $535(73)$ & $521(56)$ \\
\hline
\end{tabular}

Note: DLPFC, Dorsolateral prefrontal cortex. RT, response time.
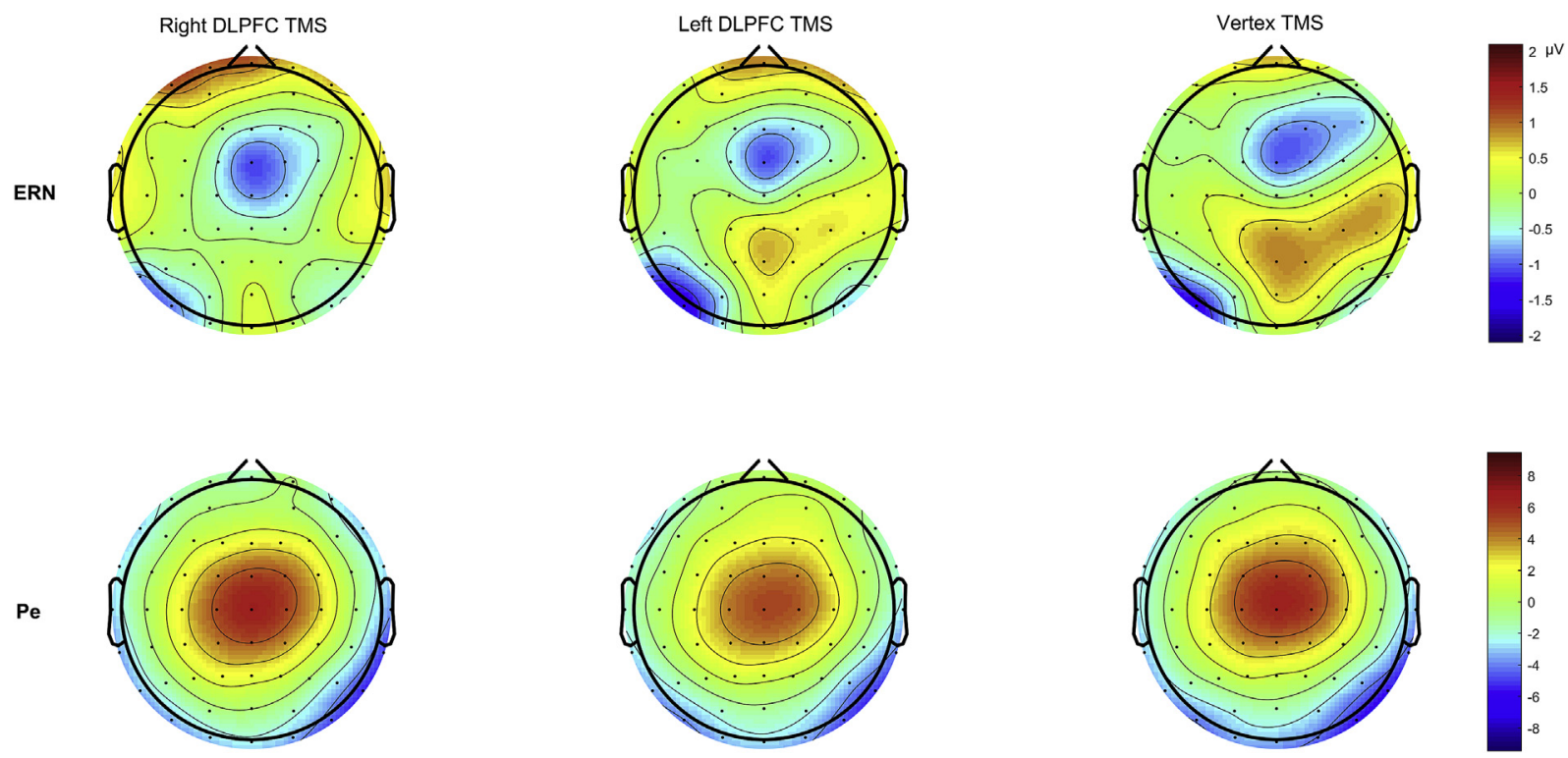

Fig. 3. Scalp topography of ERP evoked by aware errors as a function of stimulation site (right DLPFC/left DLPFC/Vertex). The topographical maps at the top of the figure show the scalp distribution of the ERN component, within a time window from $-100 \mathrm{~ms}$ to $50 \mathrm{~ms}$ before the incorrect response. The maps at the bottom of the figure show the distribution of the Pe, within a time window from $100 \mathrm{~ms}$ to $300 \mathrm{~ms}$ after the incorrect response. 

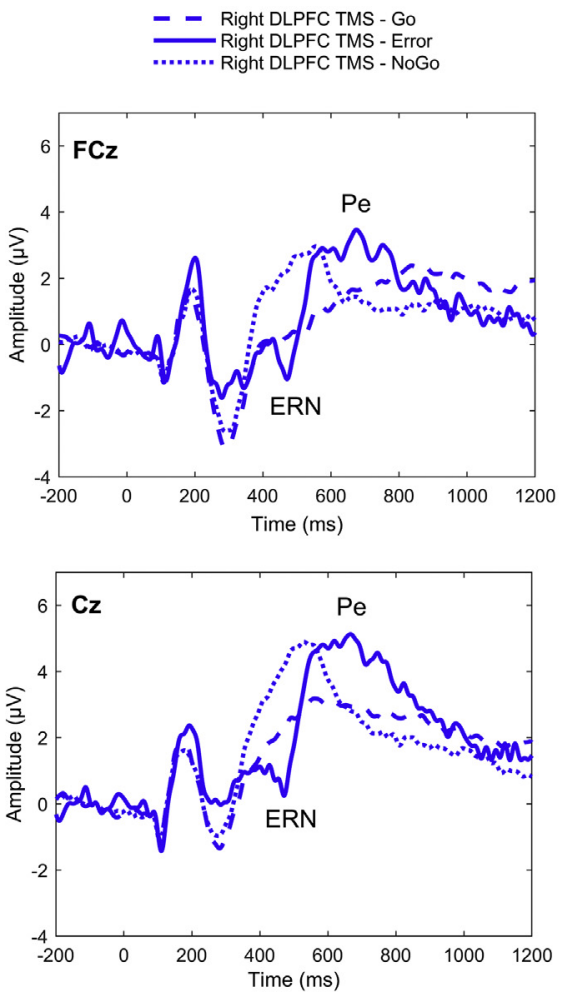

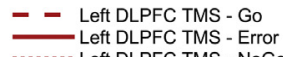

Left DLPFC TMS - Error
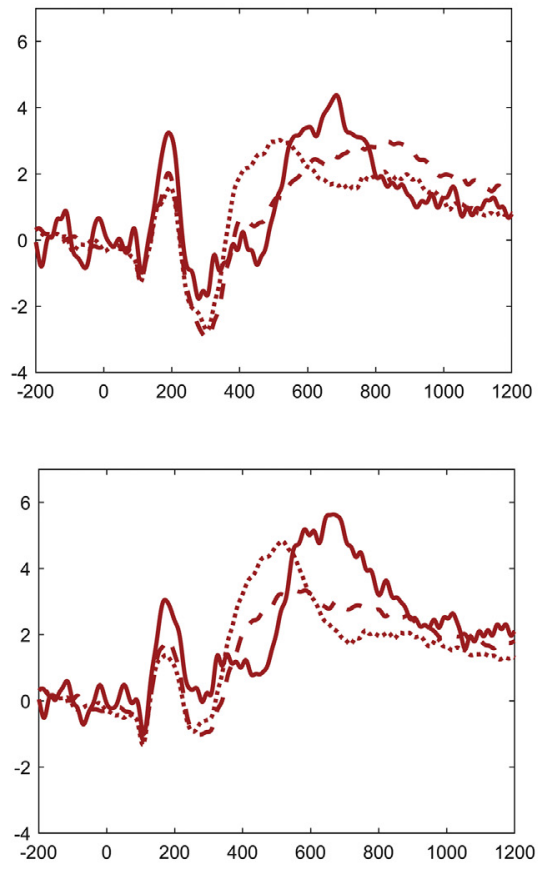

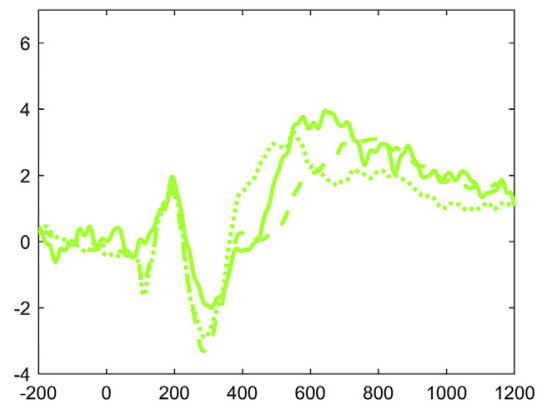

Vertex TMS - Go

Vertex TMS - Error

Vertex TMS - NoGo

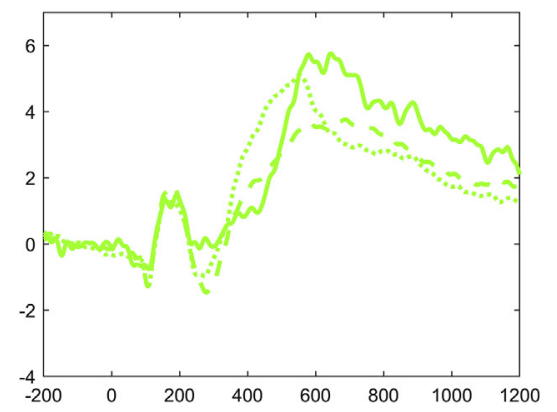

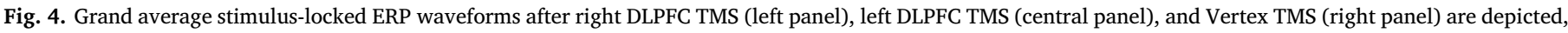

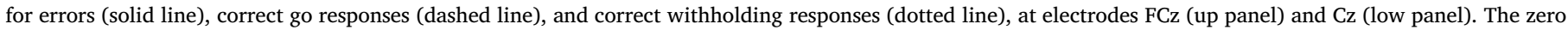
point corresponds to the stimulus onset.

revealed a main effect of trial type $(F(1,14)=31.13, p<0.0001$, partial $\left.\eta^{2}=0.69\right)$. The post-hoc comparison indicated that participants signaled more often a commission error on Stroop trials than on repeat trials $(98 \%$ vs. $71 \%)$. The analysis revealed neither a significant effect of stimulation site $\left(F(2,28)=0.02, p=0.982\right.$, partial $\left.\eta^{2}=0.001\right)$ or a significant interaction $\left(F(2,28)=0.15, p=0.857\right.$, partial $\left.\eta^{2}=0.01\right)$.

PES - The mean RTs following and prior to an aware error are shown in Table 1. The 2 (go trial position) $\times 3$ (stimulation site) ANOVA showed neither a main effect of go trial position $(F(1,14)=1.38, p=0.259$, partial $\left.\eta^{2}=0.09\right)$ or stimulation site $(F(2,28)=3.09, p=0.061$, partial $\left.\eta^{2}=0.18\right)$ or interaction $(F(2,28)=2.93, \quad p=0.070$, partial $\left.\eta_{\mathrm{p}}^{2}=0.17\right)$.

\subsection{ERP results}

Electrical potentials evoked by aware errors, namely ERPs locked to incorrect responses in No-Go trials (i.e., Stroop and repeat conditions), were analyzed. The grand-average waveforms of error-locked ERPs (Fig. 2) clearly revealed the presence of a negative deflection around response (ERN) and a following, more pronounced, positive deflection (Pe).

To test the effect of TMS on the ERN and Pe components, the ERP amplitude in error trials was compared across stimulation sites in two time windows: around button press (from -100 to $50 \mathrm{~ms}$ ) and after button press (from 100 to $300 \mathrm{~ms}$ ), respectively. No amplitude differences across stimulated sites emerged in the time-window corresponding to the ERN. On the other hand, amplitude differences across stimulation sites emerged in the time-window corresponding to the Pe component. Namely, TMS over the left DLPFC produced a reduction of the Pe amplitude evoked by incorrect button press compared to the Pe evoked after TMS over the right DLPFC and over the Vertex (Fig. 2). Specifically, the cluster mass permutation test on point-by-point $t$ scores revealed that the Pe amplitude after left DLPFC stimulation was significantly lower than the Pe amplitude after the right DLPFC stimulation from 145 to $248 \mathrm{~ms}$ over Cz, and relative to the Pe amplitude after Vertex stimulation from 193 to $254 \mathrm{~ms}$ on Cz and from 189 to $246 \mathrm{~ms}$ on FCz.

Error-related potentials were further compared to correct Go-response potentials. These ERPs showed very attenuated or almost absent deflections, shifted in time (anticipated) relative to error-related components (Fig. 2). The statistical analyses confirmed that the ERN evoked by incorrect trials was significantly larger (more negative) than the ERP amplitude in corresponding time-points in correct Go trials, namely, in the right DLPFC session (FCz: from -33 to $37 \mathrm{~ms}$; Cz: from -78 to $45 \mathrm{~ms}$ ), in the left DLPFC session (FCz: from -55 to $35 \mathrm{~ms}$; Cz: from -76 to $51 \mathrm{~ms}$ ), and in the Vertex session (Cz: from -66 to $23 \mathrm{~ms}$ ). Likewise, the Pe was substantially larger in the incorrect trials compared to correct Go responses in the right stimulation session (FCZ: from 78 to $234 \mathrm{~ms}$; Cz: from 78 to $300 \mathrm{~ms}$ ), in the left stimulation session (FCz: from 88 to $191 \mathrm{~ms}$; Cz: from 88 to $300 \mathrm{~ms}$ ), and in the Vertex stimulation session (FCz: from 102 to $209 \mathrm{~ms}$; Cz: from 96 to $283 \mathrm{~ms}$ ). These effects were replicated when the same number of correct-response epochs were randomly selected to match the number of errors.

The potentials measured by subtracting point-by-point ERP voltage in Go epochs from ERP voltage in error epochs were significantly different between left and right stimulation sessions, in the Pe timewindow. Specifically, this difference potential was attenuated after the left DLPFC session compared to right DLPFC session, from 139 to $258 \mathrm{~ms}$, over Cz. Given the shifting in time between error and correctresponse ERPs one should take these differences cautiously.

TMS did not yield significant modulation on Go-response locked ERPs, apart from a small amplitude difference between left and right DLPFC stimulation, at early time points, from 100 to $166 \mathrm{~ms}$, over Cz. The number of errors did not significantly correlate with the ERP mean amplitude in the Pe time-window (right DLPFC: $\rho(15)=-0.021$, $p=0.939 ; \quad$ left $\quad$ DLPFC: $\rho(15)=-0.154, \quad p=0.583$; Vertex: $\rho(15)=0.041, p=0.884)$. 
The Fig. 4 represents ERPS locked to stimulus onset in error, correct Go and correct withhold trials. Here, error-related components are also appreciable, although epochs are not temporally aligned to response, and distinguishable from stimulus evoked N2 and P3 components.

\section{Discussion}

The aim of the present study was to examine the contribution of the DLPFC on error-related processes through a combined TMS-EEG approach, which provides a more fine-grained investigation of TMS-induced neural modulations (Miniussi and Thut, 2010; Thut and PascualLeone, 2010). While previous studies have mainly focused on examining the role of the right DLPFC and the left DLPFC in separated studies (Mansouri et al., 2016; Rollnik et al., 2004), we compared the contribution of these regions within the same experiment.

The main finding that emerged from the study was an association between the stimulation of the left DLPFC and the amplitude of a wellknown post-error ERP component, the Pe. Specifically, a repetitive inhibitory magnetic stimulation over this prefrontal brain region led to diminished amplitude of the Pe potential relative to conditions in which either the right DLPFC or the control site (the Vertex) were inhibited. This result is partially consistent with a previous study that found a reduction of the Pe amplitude following cathodal tDCS over the medial frontal cortex (Bellaïche et al., 2013). However, in that previous study was not possible to establish which hemisphere played a role in modulating the Pe for two main reasons: the poor spatial resolution of tDCS and, most importantly, the central position of the active electrode, namely FCz. In our study, for the first time, we demonstrated a causal role of the left DLPFC on error-related processes. Specifically, by comparing the neurophysiological activity modulated by TMS between the right and left DLPFC stimulation, we demonstrated that only the left DLPFC was involved in a circuit that controls error processing and contributes to the modulation of the Pe. At the same time, we should also recognize the possibility that rTMS could have modulated areas functionally connected to the left DLPFC. However, regardless of this consideration, the present study strongly supports the implication of the left DLPFC as a crucial node implicated in the generation of the Pe.

According to previous ERP findings, the Pe component has been thought to reflect multiple functions, such as the conscious detection of an error (Endrass et al., 2007; Hoonakker et al., 2016; Murphy et al., 2012; O'Connell et al., 2007), adaptive adjustments following an error (Overbeek et al., 2005), or the motivational significance attribute to an error (Ridderinkhof et al., 2009). If this component reflects the conscious error detection, after the reduction of the Pe amplitude we should have obtained lower percentages of aware errors, which was not the case. However, even if rTMS on the left DLPFC reduced the mean amplitude of the Pe, it could not be sufficient to disrupt error awareness. In line with this explanation, a recent study showed that the Pe would reflect the strength of accumulated evidence about error commission (Murphy et al., 2012). Thus, the Pe amplitude may reflect somehow the level of error awareness. Furthermore, several studies assume that the emergence of error awareness can be conceptualized as a decision process, in which awareness about an error is achieved after that a sufficient evidence of initial error commission has been accumulated up to reach a decisional threshold (Steinhauser and Yeung, 2010; Steinhauser et al., 2008). Compatibly with this account, the Pe amplitude may represent the electrophysiological marker of this evidence-based accumulation process. In our study, the decisional threshold (error awareness) was reached anyway, despite the Pe reduction induced by means of rTMS. Thus, although rTMS on the left DLPFC modulated the mean amplitude of the Pe, it was not sufficient to interfere with error awareness.

The stimulation of the DLPFC did not induce changes on the ERN amplitude. Although speculative, we might infer that this ERP component is generated from other brain locations, likely medial frontal areas, such as the ACC (Ito et al., 2003; Van Veen and Carter, 2002).
The fact that in this study the maximum ERN amplitude was reached immediately before the response time is in line with some previous evidence (e.g., Gehring et al., 2012) and might depend on the nature of the task itself (i.e., the Go/No-Go). Indeed, participants must withhold a simple/one-choice button press. Therefore, an error on such inhibition process might occur before motor response, (i.e., the actual button press). A second explanation may be the strong dependence of the ERN on the actual time-locking event (i.e. the response), which may have spread the ERN amplitudes over time. Perhaps electromyographylocked epochs would have led to more robust findings.

Neither the right nor the left DLPFC stimulation had an influence on the rate of errors in the present experiment. This partially replicates previous null observation (e.g., Rollnik et al., 2004).

The absence of PES could be explained by task requirements. Indeed, participants had to signal the commission error by an additional button press, as fast as possible after the wrong button press. This additional motor requirement would have interfered with the creation of a proper PES.

An unexpected finding consists on the fact that stimulations did not yield effects on the number of aware errors. This observation contrasts with previous findings in which the DLPFC appeared implicated in error awareness (Harty et al., 2014; Masina et al., 2018). However, in these studies a different stimulation protocol (Masina et al., 2018) or a different NIBS technique, namely tDCS (Harty et al., 2014), was employed. Thus, the choice of the stimulation paradigm or NIBS technique may contribute to different effects. Further TMS-EEG studies should possibly consider an on-line stimulation protocol, where the DLPFC is targeted soon after incorrect response press, in order to verify the intervention of the left DLPFC on the Pe.

In our study we should recognized some limitations. We found that an electrophysiological index, but not behavioral outcomes, was modulated by an inhibitory TMS protocol. The discrepancy between electrophysiological and behavioral measures confirms the importance of the combined use of TMS with other neuroimaging techniques in order to obtain more sensitive information. Given the Go/No-Go nature of the task and the relationship between the ERN and impulsivity (Taylor et al., 2018), it is advisable that future investigations would also take into account the effect of impulsive behavior on error processing.

Overall, the present TMS-EEG findings remark the role of the left DLPFC in error processing and encourage future investigations to better understand the functional contribution of lateral prefrontal areas along with the well-known role of medial frontal areas.

\section{CRediT authorship contribution statement}

Fabio Masina: Conceptualization, Investigation, Writing - original draft, Data curation, Formal analysis, Methodology, Project administration, Visualization, Writing - review \& editing. Vincenza Tarantino: Conceptualization, Investigation, Writing - original draft, Data curation, Formal analysis, Methodology, Software, Validation, Visualization, Writing - review \& editing. Antonino Vallesi: Investigation, Supervision, Resources, Validation, Writing - review \& editing. Daniela Mapelli: Investigation, Supervision, Resources, Writing - review \& editing.

\section{Acknowledgments}

We thank Andrea Castellano and Francesca Scarpata for helping in data collection.

\section{References}

Bellaïche, L., Asthana, M., Ehlis, A.-C., Polak, T., Herrmann, M.J., 2013. The modulation of error processing in the medial frontal cortex by transcranial direct current stimulation. Neurosci. J. https://doi.org/10.1155/2013/187692.

Bonini, F., Burle, B., Lieǵeois-Chauvel, C., Reǵis, J., Chauvel, P., Vidal, F., 2014. Action 
monitoring and medial frontal cortex: leading role of supplementary motor area. Science 343, 888-891. https://doi.org/10.1126/science.1247412.

Botvinick, M.M., Braver, T.S., Barch, D.M., Carter, C.S., Cohen, J.D., 2001. Conflict monitoring and cognitive control. Psychol. Rev. 108, 624-652. https://doi.org/10. 1037/0033-295X.108.3.624.

Brázdil, M., Roman, R., Falkenstein, M., Daniel, P., Jurák, P., Rektor, I., 2002. Error processing - evidence from intracerebral ERP recordings. Exp. Brain Res. 146, 460-466. https://doi.org/10.1007/s00221-002-1201-y.

Chaumon, M., Bishop, D.V.M., Busch, N.A., 2015. A practical guide to the selection of independent components of the electroencephalogram for artifact correction. J. Neurosci. Methods 250, 47-63. https://doi.org/10.1016/j.jneumeth.2015.02.025.

Chen, R., Classen, J., Gerloff, C., Celnik, P., Wassermann, E.M., Hallett, M., Cohen, L.G., 1997. Depression of motor cortex excitability by low-frequency transcranial magnetic stimulation. Neurology 48, 1398-1403. https://doi.org/10.1212/WNL.48.5.1398.

Cieslik, E.C., Zilles, K., Caspers, S., Roski, C., Kellermann, T.S., Jakobs, O., Langner, R., Laird, A.R., Fox, P.T., Eickhoff, S.B., 2013. Is there one DLPFC in cognitive action control? Evidence for heterogeneity from Co-activation-based parcellation. Cerebr. Cortex 23, 2677-2689. https://doi.org/10.1093/cercor/bhs256.

Coleman, J.R., Watson, J.M., Strayer, D.L., 2018. Working memory capacity and task goals modulate error-related ERPs. Psychophysiology 55. https://doi.org/10.1111/ psyp. 12805.

Danielmeier, C., Ullsperger, M., 2011. Post-error adjustments. Front. Psychol. https:// doi.org/10.3389/fpsyg.2011.00233.

Debener, S., 2005. Trial-by-Trial coupling of concurrent electroencephalogram and functional magnetic resonance imaging identifies the dynamics of performance monitoring. J. Neurosci. https://doi.org/10.1523/jneurosci.3286-05.2005.

Delorme, A., Makeig, S., 2004. EEGLAB: an open source toolbox for analysis of single-trial EEG dynamics including independent component analysis. J. Neurosci. Methods 134, 9-21. https://doi.org/10.1016/j.jneumeth.2003.10.009.

Delorme, A., Sejnowski, T., Makeig, S., 2007. Enhanced detection of artifacts in EEG data using higher-order statistics and independent component analysis. Neuroimage. https://doi.org/10.1016/j.neuroimage.2006.11.004.

Di Gregorio, F., Maier, M.E., Steinhauser, M., 2018. Errors can elicit an error positivity in the absence of an error negativity: evidence for independent systems of human error monitoring. Neuroimage 172, 427-436. https://doi.org/10.1016/j.neuroimage. 2018.01.081.

Dutilh, G., Van Ravenzwaaij, D., Nieuwenhuis, S., Van der Maas, H.L.J., Forstmann, B.U., Wagenmakers, E.J., 2012. How to measure post-error slowing: a confound and a simple solution. J. Math. Psychol. 56, 208-216. https://doi.org/10.1016/j.jmp. 2012. 04.001.

Endrass, T., Reuter, B., Kathmann, N., 2007. ERP correlates of conscious error recognition: aware and unaware errors in an antisaccade task. Eur. J. Neurosci. 26 1714-1720. https://doi.org/10.1111/j.1460-9568.2007.05785.x.

Falkenstein, M., Hohnsbein, J., Hoormann, J., Blanke, L., 1991. Effects of crossmodal divided attention on late ERP components. II. Error processing in choice reaction tasks. Electroencephalogr. Clin. Neurophysiol. 78, 447-455. https://doi.org/10. 1016/0013-4694(91)90062-9.

Falkenstein, M., Hoormann, J., Christ, S., Hohnsbein, J., 2000. ERP components on reaction errors and their functional significance: a tutorial. Biol. Psychol. 51, 87-107. https://doi.org/10.1016/S0301-0511(99)00031-9.

Gehring, W.J., Coles, M.G.H., Meyer, D.E., Donchin, E., 1990. The error-related negativity: an event-related brain potential accompanying errors. Psychophysiology 27, 34. https://doi.org/10.1111/j.1469-8986.1990.tb02374.x.

Gehring, W.J., Goss, B., Coles, M.G.H., Meyer, D.E., Donchin, E., 1993. A neural system for error detection and compensation. Psychol. Sci. 4, 385-390. https://doi.org/10. 1111/j.1467-9280.1993.tb00586.x.

Gehring, W.J., Knight, R.T., 2000. Prefrontal-cingulate interactions in action monitoring Nat. Neurosci. 3, 516-520. https://doi.org/10.1038/74899.

Gehring, W.J., Liu, Y., Orr, J.M., Carp, J., 2012. The error-related negativity (ERN/Ne) In: The Oxford Handbook of Event-Related Potential Components, . https://doi.org/ 10.1093/oxfordhb/9780195374148.013.0120.

Groppe, D.M., Urbach, T.P., Kutas, M., 2011. Mass univariate analysis of event-related brain potentials/fields I: a critical tutorial review. Psychophysiology. https://doi. org/10.1111/j.1469-8986.2011.01273.x.

Harty, S., Robertson, I.H., Miniussi, C., Sheehy, O.C., Devine, C. a, McCreery, S. O'Connell, R.G., 2014. Transcranial direct current stimulation over right dorsolateral prefrontal cortex enhances error awareness in older age. J. Neurosci. 34, 3646-3652. https://doi.org/10.1523/JNEUROSCI.5308-13.2014.

Herrmann, M.J., Römmler, J., Ehlis, A.C., Heidrich, A., Fallgatter, A.J., 2004. Source localization (LORETA) of the error-related-negativity (ERN/Ne) and positivity (Pe). Cogn. Brain Res. 20, 294-299. https://doi.org/10.1016/j.cogbrainres.2004.02.013.

Hester, R., Foxe, J.J., Molholm, S., Shpaner, M., Garavan, H., 2005. Neural mechanisms involved in error processing: a comparison of errors made with and without awareness. Neuroimage 27, 602-608. https://doi.org/10.1016/j.neuroimage.2005.04.035.

Hester, R., Nandam, L.S., O'Connell, R.G., Wagner, J., Strudwick, M., Nathan, P.J., Mattingley, J.B., Bellgrove, M.A., 2012. Neurochemical enhancement of conscious error awareness. J. Neurosci. https://doi.org/10.1523/JNEUROSCI.4052-11.2012.

Hester, R., Nestor, L., Garavan, H., 2009. Impaired error awareness and anterior cingulate cortex hypoactivity in chronic cannabis users. Neuropsychopharmacology 34 , 2450-2458. https://doi.org/10.1038/npp.2009.67.

Hoerold, D., Pender, N.P., Robertson, I.H., 2013. Metacognitive and online error awareness deficits after prefrontal cortex lesions. Neuropsychologia 51, 385-391. https://doi.org/10.1016/j.neuropsychologia.2012.11.019.

Holroyd, C.B., Coles, M.G.H., 2002. The neural basis of human error processing: reinforcement learning, dopamine, and the error-related negativity. Psychol. Rev. 109, 679-709. https://doi.org/10.1037//0033-295X.109.4.679.
Hoonakker, M., Doignon-Camus, N., Bonnefond, A., 2016. Performance monitoring mechanisms activated before and after a response: a comparison of aware and unaware errors. Biol. Psychol. https://doi.org/10.1016/j.biopsycho.2016.08.009.

Iannaccone, R., Hauser, T.U., Staempfli, P., Walitza, S., Brandeis, D., Brem, S., 2015. Conflict monitoring and error processing: new insights from simultaneous EEG-fMRI Neuroimage 105, 395-407. https://doi.org/10.1016/j.neuroimage.2014.10.028.

Ito, S., Stuphorn, V., Brown, J.W., Schall, J.D., 2003. Performance monitoring by the anterior cingulate cortex during saccade countermanding. Science 302, 120-122. https://doi.org/10.1126/science.1087847.

Kappenman, E.S., Luck, S.J., 2012. The oxford handbook of event-related potential components, the oxford handbook of event-related potential components. https:// doi.org/10.1093/oxfordhb/9780195374148.001.0001.

Laming, D.R.J., 1968. Information theory of choice-reaction times. Inf. theory choicereaction times 14, 172. https://doi.org/10.1080/1461670X.2011.557559.

Mansouri, F.A., Fehring, D.J., Feizpour, A., Gaillard, A., Rosa, M.G.P., Rajan, R., Jaberzadeh, S., 2016. Direct current stimulation of prefrontal cortex modulates errorinduced behavioral adjustments. Eur. J. Neurosci. 44, 1856-1869. https://doi.org/ 10.1111/ejn.13281.

Masina, F., Vallesi, A., Di Rosa, E., Semenzato, L., Mapelli, D., 2018. Possible role of dorsolateral prefrontal cortex in error awareness: single-pulse TMS evidence. Front. Neurosci. 12. https://doi.org/10.3389/fnins.2018.00179.

Miniussi, C., Thut, G., 2010. Combining TMS and EEG offers new prospects in cognitive neuroscience. Brain Topogr. https://doi.org/10.1007/s10548-009-0083-8.

Murphy, P.R., Robertson, I.H., Allen, D., Hester, R., O'Connell, R.G., 2012. An electrophysiological signal that precisely tracks the emergence of error awareness. Front. Hum. Neurosci. 6, 1-16. https://doi.org/10.3389/fnhum.2012.00065.

Niessen, E., Fink, G.R., Hoffmann, H.E.M., Weiss, P.H., Stahl, J., 2017. Error detection across the adult lifespan: electrophysiological evidence for age-related deficits. Neuroimage. https://doi.org/10.1016/j.neuroimage.2017.03.015.

Nieuwenhuis, S., Ridderinkhof, K.R., Blom, J., Band, G.P.H., Kok, A., 2001. Error-related brain potentials are differentially related to awareness of response errors: evidence from an antisaccade task. Psychophysiology 38, 752-760. https://doi.org/10.1111/ 1469-8986.3850752.

Notebaert, W., Houtman, F., Opstal, F. Van, Gevers, W., Fias, W., Verguts, T., 2009. Post error slowing: an orienting account. Cognition 111, 275-279. https://doi.org/10. 1016/j.cognition.2009.02.002.

O'Connell, R.G., Bellgrove, M.A., Dockree, P.M., Lau, A., Hester, R., Garavan, H., Fitzgerald, M., Foxe, J.J., Robertson, I.H., 2009. The neural correlates of deficient error awareness in attention-deficit hyperactivity disorder (ADHD). Neuropsychologia 47, 1149-1159. https://doi.org/10.1016/j.neuropsychologia. 2009.01.011.

O'Connell, R.G., Dockree, P.M., Bellgrove, M.A., Kelly, S.P., Hester, R., Garavan, H., Robertson, I.H., Foxe, J.J., 2007. The role of cingulate cortex in the detection of errors with and without awareness: a high-density electrical mapping study. Eur. J. Neurosci. 25, 2571-2579. https://doi.org/10.1111/j.1460-9568.2007.05477.x.

Olvet, D.M., Hajcak, G., 2008. The error-related negativity (ERN) and psychopathology: toward an endophenotype. Clin. Psychol. Rev. https://doi.org/10.1016/j.cpr.2008. 07.003 .

Overbeek, T.J.M., Nieuwenhuis, S., Ridderinkhof, K.R., 2005. Dissociable components of error processing. J. Psychophysiol. 19, 319-329. https://doi.org/10.1027/0269 8803.19.4.319.

Pontifex, M.B., Scudder, M.R., Brown, M.L., O'Leary, K.C., Wu, C.T., Themanson, J.R., Hillman, C.H., 2010. On the number of trials necessary for stabilization of errorrelated brain activity across the life span. Psychophysiology 47, 767-773. https:// doi.org/10.1111/j.1469-8986.2010.00974.x.

Qiu, L., Su, J., Ni, Y., Bai, Y., Zhang, X., Li, X., Wan, X., 2018. The neural system of metacognition accompanying decision-making in the prefrontal cortex. PLoS Biol. https://doi.org/10.1371/journal.pbio.2004037.

Rabbitt, P.M., 1966. Errors and error correction in choice-response tasks. J. Exp. Psychol. 71, 264-272. https://doi.org/10.1037/h0022853.

Reinhart, R.M.G., Woodman, G.F., 2014. Causal control of medial-frontal cortex governs electrophysiological and behavioral indices of performance monitoring and learning. J. Neurosci. https://doi.org/10.1523/JNEUROSCI.5421-13.2014.

Ridderinkhof, K.R., Ramautar, J.R., Wijnen, J.G., 2009. To PEor not to PE: a P3-like ERP component reflecting the processing of response errors. Psychophysiology 46 , 531-538. https://doi.org/10.1111/j.1469-8986.2009.00790.x.

Rollnik, J.D., Schröder, C., Rodríguez-Fornells, A., Kurzbuch, A.R., Däuper, J., Möller, J., Münte, T.F., 2004. Functional lesions and human action monitoring: combining repetitive transcranial magnetic stimulation and event-related brain potentials. Clin. Neurophysiol. 115, 145-153. https://doi.org/10.1016/j.clinph.2003.05.001.

Rossi, S., Hallett, M., Rossini, P.M., Pascual-Leone, A., 2011. Screening questionnaire before TMS: an update. Clin. Neurophysiol. 122, 1686. https://doi.org/10.1016/j. clinph.2010.12.037.

Rossi, S., Hallett, M., Rossini, P.M., Pascual-Leone, A., Avanzini, G., Bestmann, S., Berardelli, A., Brewer, C., Canli, T., Cantello, R., Chen, R., Classen, J., Demitrack, M., Di Lazzaro, V., Epstein, C.M., George, M.S., Fregni, F., Ilmoniemi, R., Jalinous, R. Karp, B., Lefaucheur, J.P., Lisanby, S., Meunier, S., Miniussi, C., Miranda, P., Padberg, F., Paulus, W., Peterchev, A., Porteri, C., Provost, M., Quartarone, A., Rotenberg, A., Rothwell, J., Ruohonen, J., Siebner, H., Thut, G., Valls-Solè, J., Walsh, V., Ugawa, Y., Zangen, A., Ziemann, U., 2009. Safety, ethical considerations, and application guidelines for the use of transcranial magnetic stimulation in clinical practice and research. Clin. Neurophysiol. https://doi.org/10.1016/j.clinph.2009.08.016.

Sellaro, R., van Leusden, J.W.R., Tona, K.-D., Verkuil, B., Nieuwenhuis, S., Colzato, L.S., 2015. Transcutaneous vagus nerve stimulation enhances post-error slowing. J. Cogn. Neurosci. 27, 2126-2132. https://doi.org/10.1162/jocn_a_00851.

Sokhadze, E.M., Baruth, J.M., Sears, L., Sokhadze, G.E., El-Baz, A.S., Casanova, M.F., 
2012. Prefrontal neuromodulation using rTMS improves error monitoring and correction function in autism. Appl. Psychophysiol. Biofeedback 37, 91-102. https://doi. org/10.1007/s10484-012-9182-5.

Sokhadze, E.M., El-Baz, A.S., Sears, L.L., Opris, I., Casanova, M.F., 2014. rTMS neuromodulation improves electrocortical functional measures of information processing and behavioral responses in autism. Front. Syst. Neurosci. 8. https://doi.org/10. 3389/fnsys.2014.00134.

Steinhauser, M., Maier, M., Hubner, R., 2008. Modeling behavioral measures of error detection in choice tasks: response monitoring versus conflict monitoring. J. Exp. Psychol. Hum. Percept. Perform. 34, 158-176. https://doi.org/10.1037/0096-1523. 34.1.158.

Steinhauser, M., Yeung, N., 2010. Decision processes in human performance monitoring. J. Neurosci. 30, 15643-15653. https://doi.org/10.1523/JNEUROSCI.1899-10.2010.

Taylor, J.B., Visser, T.A.W., Fueggle, S.N., Bellgrove, M.A., Fox, A.M., 2018. The errorrelated negativity (ERN) is an electrophysiological marker of motor impulsiveness on the Barratt Impulsiveness Scale (BIS-11) during adolescence. Dev. Cogn. Neurosci. https://doi.org/10.1016/j.dcn.2018.01.003.

Thut, G., Pascual-Leone, A., 2010. A review of combined TMS-EEG studies to characterize lasting effects of repetitive TMS and assess their usefulness in cognitive and clinical neuroscience. Brain Topogr. https://doi.org/10.1007/s10548-009-0115-4.

Ullsperger, M., Danielmeier, C., Jocham, G., 2014. Neurophysiology of performance monitoring and adaptive behavior. Physiol. Rev. 94, 35-79. https://doi.org/10. 1152/physrev.00041.2012.

Ullsperger, M., Von Cramon, D.Y., 2001. Subprocesses of performance monitoring: a dissociation of error processing and response competition revealed by event-related fMRI and ERPs. Neuroimage 14, 1387-1401. https://doi.org/10.1006/nimg.2001. 0935.

van Boxtel, G.J.M., van der Molen, M.W., Jennings, J.R., 2005. Differential involvement of the anterior cingulate cortex in performance monitoring during a stop-signal task. J. Psychophysiol. https://doi.org/10.1027/0269-8803.19.1.1.

Van Veen, V., Carter, C.S., 2002. The anterior cingulate as a conflict monitor: FMRI and ERP studies. Physiol. Behav. 77, 477-482. https://doi.org/10.1016/S0031-9384(02) 00930-7.

Veen, V. van, Carter, C.S., 2002. The timing of action-monitoring processes in the anterior cingulate cortex. J. Cogn. Neurosci. 14, 593-602. https://doi.org/10.1162/ 08989290260045837.

Wessel, J.R., Klein, T.A., Ott, D.V.M., Ullsperger, M., 2014. Lesions to the prefronta performance-monitoring network disrupt neural processing and adaptive behaviors after both errors and novelty. Cortex. https://doi.org/10.1016/j.cortex.2013.09.002.

Widmann, A., Schröger, E., Maess, B., 2015. Digital filter design for electrophysiological data - a practical approach. J. Neurosci. Methods. https://doi.org/10.1016/j. jneumeth.2014.08.002. 